ACHD is required to differentiate low risk from high risk patients. The role of NOACs in this group of patients is unclear and needs further evidence. 1 patient had a stroke on a NOAC despite being compliant.

\section{Stable IHD/Prevention/Hypertension/Lipids}

\section{PROPHYLAXIS OF VENOUS THROMBOEMBOLISM: ENSURING APPROPRIATE PRESCRIBING AND RELIABLE DATA COLLECTION}

${ }^{1}$ Oliver Ziff*, ${ }^{2}$ Paul R Carter, ${ }^{2}$ Ben Coombs, ${ }^{2}$ Gianpietro Signorini, ${ }^{2}$ Seonaird Pye, ${ }^{2}$ Chris Laing, ${ }^{2}$ Ameet Bakhai. 'University of Birmingham; ${ }^{2}$ Royal Free London NHS Foundation Trust; *Presenting Author

\subsection{6/heartjnl-2016-309890.76}

Background Venous thromboembolism (VTE) is the most common preventable cause of death with safe and effective prevention measures available. Inpatients at high risk for VTE often fail to be provided prophylaxis despite clear guidelines and this mismatch between VTE prophylaxis and thromboembolic risk is a major issue for clinicians. We sought to clarify compliance of practice with the NICE quality standard and to assess accuracy of the Commissioning for Quality and Innovation (CQUIN) VTE form.

Methods A point prevalence study was performed involving a comprehensive review of 100 consecutive patients at Barnet Hospital. We assessed compliance against the NICE guideline 92 by ensuring appropriate assessment of VTE and bleeding risk, clarifying that those where the VTE risk outweighs the bleeding risk are offered prophylaxis. We also assessed the accuracy of VTE risk assessment form completion using validated bleeding risk (HASBLED) and VTE risk (Wells) scores. We implemented technical improvements in the VTE risk assessment form; medical education through mandatory completion of VTE eLearning modules; and involvement of the MDT through grand round discussions. Following implementation, a reaudit of 50 patients was performed.

Results In 100 patients assessed, mean age was 71 years, 55 were male, with mean weight $74.8 \mathrm{~kg}$; see Table 1 . Based on the drug chart, VTE prophylaxis was appropriately offered in $69 \%$ of patients; see Figure 1. The VTE risk assessment form was accurately completed in $57 \%$ for VTE risk status, in $69 \%$ for bleeding risk, and in $69 \%$ for VTE prophylaxis prescribed. After implementations, the reaudit revealed VTE prophylaxis was appropriate in $88 \%$, VTE risk assessment form was accurate in $78 \%$ for VTE risk status, $90 \%$ for bleeding risk, and $89 \%$ for VTE prophylaxis prescribed. The section of the drug chart devoted to VTE risk assessment was poorly utilised; $21 \%$ in the initial audit and $18 \%$ in the reaudit.

Conclusion Whilst single centre and modest in size, this robust, complete audit demonstrated compliance with VTE prophylaxis guidelines was poor, for both risk assessment and appropriate prescribing. Technical improvements to the VTE form, educating trainees and involving the MDT significantly improved compliance with guidelines. Future work is required to overcome accuracy issues of form completion ensuring patients receive optimal care.
Abstract 76 Table 1 Characteristics of the participants by initial audit and reaudit

\begin{tabular}{lll}
\hline Baseline Characteristic & Initial Audit & Reaudit \\
\hline $\mathrm{N}$ & 100 & 50 \\
Mean Age (years) \pm SD & $71 \pm 18$ & $72 \pm 19$ \\
Male (\%) & 55 & 36 \\
Body Weight (kg) & 74.8 & 72.9 \\
Atrial Fibrillation(\%) & 18 & 20 \\
Heart Failur (e\%) & 12 & 18 \\
Hypertension(\%) & 47 & 26 \\
Diabetes (\%) & 25 & 10 \\
Chronic Kidney Disease\% & 18 & 16 \\
\hline
\end{tabular}

$\mathrm{SD}$, standard deviation

\section{IMPACT OF CARDIOVASCULAR COMORBIDITIES ON MORTALITY AMONGST PSYCHIATRIC PATIENTS IN THE UNITED KINGDOM}

${ }^{1}$ Paul R Carter*, ${ }^{2}$ Andrew Carter, ${ }^{3}$ Jennifer Reynolds, ${ }^{2}$ Hardeep Uppal, ${ }^{4}$ Suresh Chandran, ${ }^{2}$ Rahul Potluri. ${ }^{1}$ Royal Free London NHS Foundation Trust; ${ }^{2}$ ACALM Study Unit in Collaboration with Aston Medical School; ${ }^{3}$ University of Birmingham; ${ }^{4}$ Department of Acute Medicine, North Western Deanery; *Presenting Author

\subsection{6/heartjnl-2016-309890.77}

Introduction Cardiovascular disease is increasingly being associated with novel risk factors including psychiatric diseases. Although a high prevalence of cardiovascular comorbidities has been demonstrated in psychiatric patients, the impact of these on mortality are yet to be studied.

Objectives We aimed to investigate the prevalence of cardiovascular comorbidities and their contribution to mortality amongst psychiatric patient in North England, UK.

Methods Anonymous information on adult psychiatric patients was obtained from hospitals in North England, UK between $1^{\text {st }}$ January 2000 and $31^{\text {st }}$ March 2013. This data was analysed according to the ACALM (Algorithm for Comorbidities, Associations, Length of stay and Mortality) study protocol. ICD-10 and OPCS-4 codes were used to trace patients coded for psychiatric disease, demographics, prevalence of cardiovascular comorbidities and mortality data. Mortality of psychiatric patients with and without cardiovascular comorbidities were

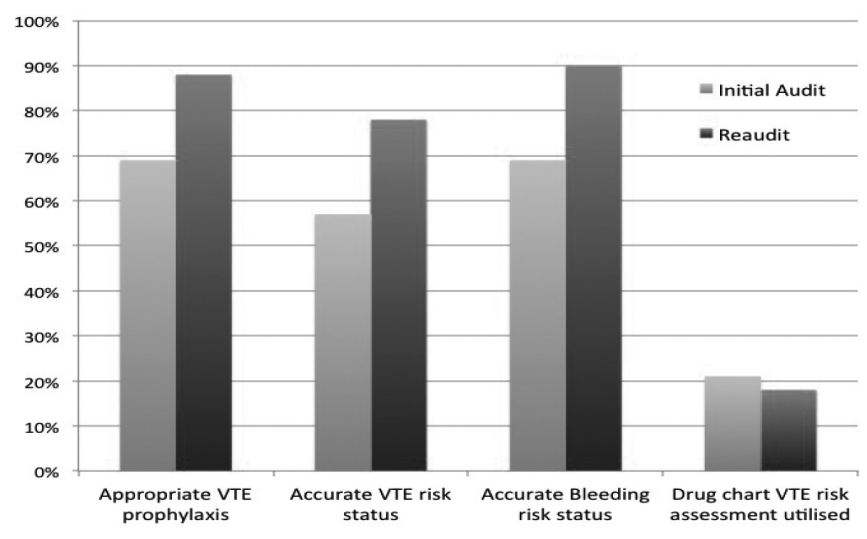

Abstract 76 Figure 1 